\title{
Quaternion Fourier Transform on Quaternion Fields and Generalizations
}

\author{
Eckhard M. S. Hitzer
}

Soli Deo Gloria

\begin{abstract}
We treat the quaternionic Fourier transform (QFT) applied to quaternion fields and investigate QFT properties useful for applications. Different forms of the QFT lead us to different Plancherel theorems. We relate the QFT computation for quaternion fields to the QFT of real signals. We research the general linear $(G L)$ transformation behavior of the QFT with matrices, Clifford geometric algebra and with examples. We finally arrive at wide-ranging non-commutative multivector FT generalizations of the QFT. Examples given are new volume-time and spacetime algebra Fourier transformations.
\end{abstract}

Mathematics Subject Classification (2000). Primary 42A38; Secondary 11R52.

Keywords. Quaternions, Fourier transform, Clifford algebra, volume-time algebra, spacetime algebra, automorphisms.

\section{Introduction}

This paper strives to deepen the understanding of the quaternionic Fourier transform (QFT) applied to quaternion fields $f: \mathbb{R}^{2} \rightarrow \mathbb{H}$, and not only to real signals $f: \mathbb{R}^{2} \rightarrow \mathbb{R}$. We research QFT properties useful for applications to partial differential equations, image processing and optimized numerical implementations. We investigate how different forms of the QFT allow to establish scalar and quaternion valued Plancherel theorems.

We show systematically how to reduce the computation for quaternion fields to the case of real signal computations, and on the other hand how results for real signals can be generalized to quaternion fields.

I thank my family and FTHD organizer S.L. Eriksson. 
The third major focus is on deriving the behavior of the QFT under $G L\left(\mathbb{R}^{2}\right)$ automorphisms. To do this we split the QFT appropriately, and work with invariant techniques of Clifford geometric algebra [1] to establish and understand the automorphism behavior. Details are brought to light by looking at the examples of stretches (dilations), reflections and rotations.

Together with isomorphisms (to Clifford subalgebras) we finally arrive at wide-ranging generalizations of the QFT. These new non-commutative multivector Fourier transforms operate on functions from domain spaces $\mathbb{R}^{m, n}$ (with $m, n \in \mathbb{N}_{0}$ ) to Clifford algebras $C l_{m, n}$ or subalgebras thereof. To demonstrate the method, we work out generalizations to volume-time and to spacetime algebra Fourier transformations, and provide some physical interpretation.

\subsection{Basic facts about Quaternions}

Gauss, Rodrigues and Hamilton 2 invented the four-dimensional quaternion algebra $\mathbb{H}$ over $\mathbb{R}$ with three imaginary units $\boldsymbol{i}, \boldsymbol{j}, \boldsymbol{k}$ and multiplication laws:

$$
\boldsymbol{i} \boldsymbol{j}=-\boldsymbol{j} \boldsymbol{i}=\boldsymbol{k}, \boldsymbol{j} \boldsymbol{k}=-\boldsymbol{k} \boldsymbol{j}=\boldsymbol{i}, \boldsymbol{k} \boldsymbol{i}=-\boldsymbol{i} \boldsymbol{k}=\boldsymbol{j}, \boldsymbol{i}^{2}=\boldsymbol{j}^{2}=\boldsymbol{k}^{2}=\boldsymbol{i} \boldsymbol{j} \boldsymbol{k}=-1 .
$$

Quaternions are isomorphic to the Clifford geometric algebra $C l_{0,2}$ of $\mathbb{R}^{0,2}$, and to the even subalgebra $C l_{3,0}^{+}$of the Clifford geometric algebra $C l_{3,0}$ of $\mathbb{R}^{3}$ :

$$
\mathbb{H} \cong C l_{0,2} \cong C l_{3,0}^{+} .
$$

$C l_{3,0}^{+}$has, with an orthonormal basis $\left\{\mathbf{e}_{1}, \mathbf{e}_{2}, \mathbf{e}_{3}\right\}$ of $\mathbb{R}^{3}$, the four dimensional basis

$$
\left\{1, \mathbf{e}_{32}=\mathbf{e}_{3} \mathbf{e}_{2}, \mathbf{e}_{13}=\mathbf{e}_{1} \mathbf{e}_{3}, \mathbf{e}_{21}=\mathbf{e}_{2} \mathbf{e}_{1}\right\}
$$

Every quaternion

$$
q=q_{r}+q_{i} \boldsymbol{i}+q_{j} \boldsymbol{j}+q_{k} \boldsymbol{k} \in \mathbb{H}, \quad q_{r}, q_{i}, q_{j}, q_{k} \in \mathbb{R}
$$

has a quaternion conjugate (corresponding to reversion in $\mathrm{Cl}_{3,0}^{+}$)

$$
\tilde{q}=q_{r}-q_{i} \boldsymbol{i}-q_{j} \boldsymbol{j}-q_{k} \boldsymbol{k},
$$

This leads to a norm of $q \in \mathbb{H}$ defined as

$$
|q|=\sqrt{q \tilde{q}}=\sqrt{q_{r}^{2}+q_{i}^{2}+q_{j}^{2}+q_{k}^{2}} .
$$

\subsection{Convenient rewriting of quaternions}

In some applications it proves convenient to replace $\boldsymbol{k}$ with $\boldsymbol{k}=\boldsymbol{i} \boldsymbol{j}$ and write a quaternion as

$$
q=q_{r}+\boldsymbol{i} q_{i}+q_{j} \boldsymbol{j}+\boldsymbol{i} q_{k} \boldsymbol{j}
$$

neatly keeping all $\boldsymbol{i}$ to the left and all $\boldsymbol{j}$ to the right of each term. A second convenient form is the split

$$
q=q_{+}+q_{-}, \quad q_{ \pm}=\frac{1}{2}(q \pm \boldsymbol{i} q \boldsymbol{j})
$$


Explicitly in real components $q_{r}, q_{i}, q_{j}, q_{k} \in \mathbb{R}$ using (1) the split (8) produces:

$$
q_{ \pm}=\left\{q_{r} \pm q_{k}+\boldsymbol{i}\left(q_{i} \mp q_{j}\right)\right\} \frac{1 \pm \boldsymbol{k}}{2}=\frac{1 \pm \boldsymbol{k}}{2}\left\{q_{r} \pm q_{k}+\boldsymbol{j}\left(q_{j} \mp q_{i}\right)\right\} .
$$

The real scalar part $q_{r}$ (grade zero selection [1] in Clifford geometric algebra)

$$
\langle q\rangle_{0}=q_{r}
$$

leads to a cyclic multiplication symmetry

$$
\langle q r s\rangle_{0}=\langle r s q\rangle_{0}, \quad \forall q, r, s \in \mathbb{H} .
$$

\subsection{Quaternion module}

For quaternion-valued functions $f, g: \mathbb{R}^{2} \rightarrow \mathbb{H}$ we can define the quaternion-valued inner product

$$
(f, g)=\int_{\mathbb{R}^{2}} f(\mathbf{x}) \tilde{g}(\mathbf{x}) d^{2} \mathbf{x}, \quad \text { with } \quad d^{2} \mathbf{x}=d x d y,
$$

with symmetric real scalar part [12]

$$
\langle f, g\rangle=\frac{1}{2}[(f, g)+(g, f)]=\int_{\mathbb{R}^{2}}\langle f(\mathbf{x}) \tilde{g}(\mathbf{x})\rangle_{0} d^{2} \mathbf{x} .
$$

Both (11) and (12) lead to the $L^{2}\left(\mathbb{R}^{2} ; \mathbb{H}\right)$-norm

$$
\|f\|=\sqrt{(f, f)}=\sqrt{\langle f, f\rangle}=\int_{\mathbb{R}^{2}}|f(\mathbf{x})|^{2} d^{2} \mathbf{x} .
$$

A quaternion module $L^{2}\left(\mathbb{R}^{2} ; \mathbb{H}\right)$ is then defined as

$$
L^{2}\left(\mathbb{R}^{2} ; \mathbb{H}\right)=\left\{f \mid f: \mathbb{R}^{2} \rightarrow \mathbb{H},\|f\|<\infty\right\} .
$$

\section{The quaternion Fourier transform}

Before defining the quaternion Fourier transform (QFT), we briefly outline its relationship with Clifford Fourier transformations.

Brackx et al. [3] extended the Fourier transform to multivector valued functiondistributions in $C l_{0, n}$ with compact support. A related applied approach for hypercomplex Clifford Fourier transformation $\sqrt{1}$ in $C l_{0, n}$ was followed by Bülow et. al. [4.

By extending the classical trigonometric exponential function $\exp (j \boldsymbol{x} * \boldsymbol{\xi})$ (where $*$ denotes the scalar product of $\boldsymbol{x} \in \mathbb{R}^{m}$ with $\boldsymbol{\xi} \in \mathbb{R}^{m}, j$ the imaginary unit) in [5, 6, McIntosh et. al. generalized the classical Fourier transform. Applied to a function of $m$ real variables this generalized Fourier transform is holomorphic in $m$ complex variables and its inverse is monogenic in $m+1$ real variables, thereby effectively extending the function of $m$ real variables to a monogenic function of $m+1$ real variables (with values in a complex Clifford algebra). This generalization has significant applications to harmonic analysis, especially to singular integrals

\footnotetext{
${ }^{1}$ This is the kind of Clifford Fourier transform to which we will refer in section 3
} 
on surfaces in $\mathbb{R}^{m+1}$. Based on this approach Kou and Qian obtained a Clifford Payley-Wigner theorem and derived Shannon interpolation of band-limitted functions using the monogenic sinc function [7, and references therein]. The Clifford Payley-Wigner theorem also allows to derive left-entire (left-monogenic in the whole $\mathbb{R}^{m+1}$ ) functions from square integrable functions on $\mathbb{R}^{m}$ with compact support.

The real $n$-dimensional volume element $i_{n}=\boldsymbol{e}_{1} \boldsymbol{e}_{2} \ldots \boldsymbol{e}_{n}$ of $C l_{n, 0}$ over the field of the reals $\mathbb{R}$ has been used in 8, 9, 10, 11 to construct and apply Clifford Fourier transformations for $n=2,3(\bmod 4)$ with kernels $\exp \left(-i_{n} \mathbf{x} * \mathbf{u}\right), \mathbf{x}, \mathbf{u} \in \mathbb{R}^{n}$. This $i_{n}$ has a clear geometric interpretation. Note that $i_{n}^{2}=-1$ for $n=2,3(\bmod 4)$.

Ell [13] defined the quaternion Fourier transform (QFT) for application to 2D linear time-invariant systems of PDEs. Ell's QFT belongs to the growing family of Clifford Fourier transformations because of (2). But the left and right placement of the exponential factors in definition 2.1 distinguishes it. Later the QFT was applied extensively to 2D image processing, including color images [12, 13, 4]. This spurred research into optimized numerical implementations [14, 15]. Ell [13] and others [12, [16 also investigated related commutative hypercomplex Fourier transforms like in the commutative subalgebra of $C l_{4,0}$ with subalgebra basis $\left\{1, \mathbf{e}_{12}, \mathbf{e}_{34}, \mathbf{e}_{1234}\right\}$,

$$
\mathbf{e}_{12}^{2}=\mathbf{e}_{34}^{2}=-1, \quad \mathbf{e}_{1234}^{2}=+1 .
$$

Definition 2.1 (Quaternion Fourier transform (QFT)). The quaternion Fourier transform $\hat{f}: \mathbb{R}^{2} \rightarrow \mathbb{H}$ of $f \in L^{2}\left(\mathbb{R}^{2} ; \mathbb{H}\right), \mathbf{x}=x \mathbf{e}_{1}+y \mathbf{e}_{2} \in \mathbb{R}^{2}$, and $\mathbf{u}=u \mathbf{e}_{1}+v \mathbf{e}_{2} \in$ $\mathbb{R}^{2}$ is defined 3 as

$$
\hat{f}(\mathbf{u})=\int_{\mathbb{R}^{2}} e^{-\boldsymbol{i x u}} f(\mathbf{x}) e^{-\boldsymbol{j} y v} d^{2} \mathbf{x} .
$$

The QFT can be inverted by

$$
f(\mathbf{x})=\frac{1}{(2 \pi)^{2}} \int_{\mathbb{R}^{2}} e^{i x u} \hat{f}(\mathbf{u}) e^{j y v} d^{2} \mathbf{u},
$$

with $d^{2} \mathbf{u}=d u d v$.

\subsection{Rewriting and splitting functions}

Let $f: \mathbb{R}^{2} \rightarrow \mathbb{H}\left(\right.$ or $\left.f \in L^{2}\left(\mathbb{R}^{2} ; \mathbb{H}\right)\right)$. Using four $\mathbb{R}^{2} \rightarrow \mathbb{R}\left(\right.$ or $\left.L^{2}\left(\mathbb{R}^{2} ; \mathbb{R}\right)\right)$ real component functions $f_{r}, f_{i}, f_{j}$, and $f_{k}$ we can decompose and rewrite $f$ with (7) as

$$
f=f_{r}+f_{i} \boldsymbol{i}+f_{j} \boldsymbol{j}+f_{k} \boldsymbol{k}=f_{r}+\boldsymbol{i} f_{i}+f_{j} \boldsymbol{j}+\boldsymbol{i} f_{k} \boldsymbol{j} .
$$

We can also split the functions $f$ [similar to $q_{ \pm}$in (8)] into

$$
f=f_{+}+f_{-}, \quad f_{+}=\frac{1}{2}(f+\boldsymbol{i} f \boldsymbol{j}), \quad f_{-}=\frac{1}{2}(f-\boldsymbol{i} f \boldsymbol{j}) .
$$

\footnotetext{
${ }^{2}$ We also assume always that $\int_{\mathbb{R}^{2}}|f(\mathbf{x})| d^{2} \mathbf{x}$ exists as well. But we do not explicitly write this condition again in the rest of the paper.

${ }^{3}$ For real signals $f \in L^{2}\left(\mathbb{R}^{2} ; \mathbb{R}\right)$ the detailed relationship of the QFT of definition 2.1 with the conventional scalar FT, i.e. with the even cos-part and the odd sin-part are given on pp. 191 and 192 of [4. With the help of (31) this can easily be extended to the full QFT of quaternion-valued $f \in L^{2}\left(\mathbb{R}^{2} ; \mathbb{H}\right)$.
} 
TABle 1. Properties of the quaternion Fourier transform (QFT) of quaternion functions (Quat. Funct.) $f, g \in L^{2}\left(\mathbb{R}^{2} ; \mathbb{H}\right)$, with $\mathbf{x}, \mathbf{u} \in \mathbb{R}^{2}$, constants $\alpha, \beta \in\left\{q \mid q=q_{r}+q_{i} \boldsymbol{i}, q_{r}, q_{i} \in \mathbb{R}\right\}$, $\alpha^{\prime}, \beta^{\prime} \in\left\{q \mid q=q_{r}+q_{j} \boldsymbol{j}, q_{r}, q_{j} \in \mathbb{R}\right\}, a, b \in \mathbb{R} \backslash\{0\}, \mathbf{x}_{0}=$ $x_{0} \mathbf{e}_{1}+y_{0} \mathbf{e}_{2}, \mathbf{u}_{0}=u_{0} \mathbf{e}_{1}+v_{0} \mathbf{e}_{2} \in \mathbb{R}^{2}$ and $m, n \in \mathbb{N}_{0}$.

\begin{tabular}{|c|c|c|}
\hline Property & Quat. Funct. & QFT \\
\hline Left linearity & $\alpha f(\mathbf{x})+\beta g(\mathbf{x})$ & $\alpha \hat{f}(\mathbf{u})+\beta \hat{g}(\mathbf{u})$ \\
\hline Right linearity & $f(\mathbf{x}) \alpha^{\prime}+g(\mathbf{x}) \beta^{\prime}$ & $\hat{f}(\mathbf{u}) \alpha^{\prime}+\hat{g}(\mathbf{u}) \beta^{\prime}$ \\
\hline x-Shift & $f\left(\mathbf{x}-\mathbf{x}_{0}\right)$ & $e^{-\boldsymbol{i} x_{0} u} \hat{f}(\mathbf{u}) e^{-\boldsymbol{j} y_{0} v}$ \\
\hline Modulation & $e^{\boldsymbol{i} x u_{0}} f(\mathbf{x}) e^{\boldsymbol{j} y v_{0}}$ & $\hat{f}\left(\mathbf{u}-\mathbf{u}_{0}\right)$ \\
\hline Dilation & $f\left(a x \mathbf{e}_{1}+b y \mathbf{e}_{2}\right)$ & $\frac{1}{|a b|} \hat{f}\left(\frac{u}{a} \mathbf{e}_{1}+\frac{v}{b} \mathbf{e}_{2}\right)$ \\
\hline Part. deriv. & $\frac{\partial^{m+n}}{\partial x^{m} \partial y^{n}} f(\mathbf{x})$ & $(\boldsymbol{i} u)^{m} \hat{f}(\mathbf{u})(\boldsymbol{j} v)^{n}$ \\
\hline Powers ${ }^{5}$ of $x, y$ & $x^{m} y^{n} f(\mathbf{x})$ & $\boldsymbol{i}^{m} \frac{\partial^{m+n}}{\partial u^{m} \partial v^{n}} \hat{f}(\mathbf{u}) \boldsymbol{j}^{n}$ \\
\hline Powers ${ }^{5}$ of $i, j$ & $\boldsymbol{i}^{m} f(\mathbf{x}) \boldsymbol{j}^{n}$ & $i^{m} \hat{f}(\mathbf{u}) j^{n}$ \\
\hline Plancherel $^{5}$ & $\langle f, g\rangle=$ & $\frac{1}{(2 \pi)^{2}}\langle\hat{f}, \hat{g}\rangle$ \\
\hline Parseval ${ }^{6}$ & $\|f\|=$ & $\frac{1}{2 \pi}\|\hat{f}\|$ \\
\hline
\end{tabular}

According to (9) the two components $f_{ \pm}$can also be rewritten as

$$
f_{ \pm}=\left\{f_{r} \pm f_{k}+\boldsymbol{i}\left(f_{i} \mp f_{j}\right)\right\} \frac{1 \pm \boldsymbol{k}}{2}=\frac{1 \pm \boldsymbol{k}}{2}\left\{f_{r} \pm f_{k}+\boldsymbol{j}\left(f_{j} \mp f_{i}\right)\right\} .
$$

As an example let us consider the split of the product of exponential functions under the QFT integral in (16). Using Euler's formula and trigonometric addition theorems the split leads to

$$
\begin{gathered}
K=e^{-\boldsymbol{i x u}} e^{-\boldsymbol{j} y v}=K_{+}+K_{-}, \\
K_{ \pm}=e^{-\boldsymbol{i}(x u \mp y v)} \frac{1 \pm \boldsymbol{k}}{2}=\frac{1 \pm \boldsymbol{k}}{2} e^{-\boldsymbol{j}(y v \mp x u)} .
\end{gathered}
$$

\subsection{Useful properties of the QFT}

We first show a new Plancherel theorem with respect to the scalar product (12).

Theorem 2.2 (QFT Plancherel). The scalar product (12) of two quaternion module functions $f, g \in L^{2}\left(\mathbb{R}^{2} ; \mathbb{H}\right)$ is given by the scalar product of the of the corresponding QFTs $\hat{f}$ and $\hat{g}$

$$
\langle f, g\rangle=\frac{1}{(2 \pi)^{2}}\langle\hat{f}, \hat{g}\rangle
$$

${ }^{4}$ Bülow [12] omits the absolute value signs for the determinant of the transformation.

${ }^{5}$ Theorems $2.4,2.5$ and 2.2

${ }^{6}$ Corollary 2.3 
Proof. For $f, g \in L^{2}\left(\mathbb{R}^{2} ; \mathbb{H}\right)$ we calculate the scalar product (12)

$$
\begin{gathered}
\langle f, g\rangle=\int_{\mathbb{R}^{2}}\langle f(\mathbf{x}) \tilde{g}(\mathbf{x})\rangle_{0} d^{2} \mathbf{x} \\
=\frac{1}{(2 \pi)^{2}} \int_{\mathbb{R}^{2}}\left\langle\int_{\mathbb{R}^{2}} e^{i u x} \hat{f}(\mathbf{u}) e^{j v y} d^{2} \mathbf{u} \tilde{g}(\mathbf{x})\right\rangle_{0} d^{2} \mathbf{x} \\
=\frac{1}{(2 \pi)^{2}} \int_{\mathbb{R}^{2}}\left\langle\hat{f}(\mathbf{u}) \int_{\mathbb{R}^{2}} e^{j v y} \tilde{g}(\mathbf{x}) e^{i u x} d^{2} \mathbf{x}\right\rangle_{0} d^{2} \mathbf{u} \\
=\frac{1}{(2 \pi)^{2}} \int_{\mathbb{R}^{2}}\left\langle\hat{f}(\mathbf{u})\left[\int_{\mathbb{R}^{2}} e^{-\boldsymbol{i u x}} g(\mathbf{x}) e^{-\boldsymbol{j} v y} d^{2} \mathbf{x}\right]^{\sim}\right\rangle_{0} d^{2} \mathbf{u} \\
=\frac{1}{(2 \pi)^{2}} \int_{\mathbb{R}^{2}}\langle\hat{f}(\mathbf{u}) \tilde{\hat{g}}(\mathbf{u})\rangle_{0} d^{2} \mathbf{u}=\frac{1}{(2 \pi)^{2}}\langle\hat{f}, \hat{g}\rangle .
\end{gathered}
$$

In the second equality of (23) we replaced $f$ with its inverse QFT expression (17). In the third equality we exchanged the order of integration and we used the cyclic symmetry (10). For the fourth equality we simply pulled the reversion outside the square brackets [...] and obtained the QFT $\hat{g}(\mathbf{u})$, which proves (22) according to (12).

For $g=f$ the Plancherel theorem 2.2 has a QFT Parseval theorem (also called Rayleigh's theorem) as a direct corollary.

Corollary 2.3 (QFT Parseval). The $L^{2}\left(\mathbb{R}^{2} ; \mathbb{H}\right)$-norm of a quaternion module function $f \in L^{2}\left(\mathbb{R}^{2} ; \mathbb{H}\right)$ is given by the $L^{2}\left(\mathbb{R}^{2} ; \mathbb{H}\right)$-norm of its QFT multiplied by $1 /(2 \pi)$

$$
\|f\|=\frac{1}{2 \pi}\|\hat{f}\| .
$$

This leads to the following observations:

- The way we obtained the Parseval theorem of cor. 2.3 is much simpler than the proofs in [12, 13.

- For two-dimensional linear time-invariant partial differential systems the Parseval theorem provides an appropriate method to measure controller performance.

- In signal processing it states that the signal energy is preserved by the QFT.

For solving PDEs with quaternionic (or real) coefficient polynomials in $x, y \in$ $\mathbb{R}^{2}$ we show the following two theorems. In this context we note again that every quaternionic (or real) coefficient polynomial in the variables $x, y \in \mathbb{R}^{2}$ can be brought into a form having factors of $i \in \mathbb{H}$ to the left side of each term and factors of $j \in \mathbb{H}$ to the right side of each term (compare (18)).

Theorem 2.4 (Powers of $x, y$ ). The QFT of a quaternion module function $x^{m} y^{n} f(\mathbf{x})$ $\in L^{2}\left(\mathbb{R}^{2} ; \mathbb{H}\right), \mathbf{x}=x \mathbf{e}_{1}+y \mathbf{e}_{2} \in \mathbb{R}^{2}, f \in L^{2}\left(\mathbb{R}^{2} ; \mathbb{H}\right), m, n \in \mathbb{N}_{0}$ is given by

$$
\widehat{x^{m} y^{n} f}(\mathbf{u})=\boldsymbol{i}^{m} \frac{\partial^{m+n}}{\partial u^{m} \partial v^{n}} \hat{f}(\mathbf{u}) \boldsymbol{j}^{n} \text {. }
$$


Proof. The proof is done by induction. It is trivial for $m=n=0$.

For $m=1, n=0$ we calculate the QFT of $\widehat{x f}$ according to (16)

$$
\begin{gathered}
\widehat{x f}(\mathbf{u})=\int_{\mathbb{R}^{2}} e^{-\boldsymbol{i} x u} x f(\mathbf{x}) e^{-\boldsymbol{j} y v} d^{2} \mathbf{x} \\
=\int_{\mathbb{R}^{2}} \boldsymbol{i} \frac{\partial}{\partial u} e^{-\boldsymbol{i} x u} f(\mathbf{x}) e^{-\boldsymbol{j} y v} d^{2} \mathbf{x} \\
=\boldsymbol{i} \frac{\partial}{\partial u} \int_{\mathbb{R}^{2}} e^{-\boldsymbol{i} x u} f(\mathbf{x}) e^{-\boldsymbol{j} y v} d^{2} \mathbf{x}=\boldsymbol{i} \frac{\partial}{\partial u} \hat{f}(\mathbf{u}) .
\end{gathered}
$$

In second equality we used $\frac{\partial}{\partial u} e^{-\boldsymbol{i} x u}=-\boldsymbol{i} x e^{-\boldsymbol{i} x u}$ and $\boldsymbol{i}(-\boldsymbol{i})=1$.

Completely analogous for $m=0, n=1$ we find

$$
\widehat{y f}(\mathbf{u})=\frac{\partial}{\partial v} \int_{\mathbb{R}^{2}} e^{-\boldsymbol{i} x u} f(\mathbf{x}) e^{-\boldsymbol{j} y v} d^{2} \mathbf{x} \boldsymbol{j}=\frac{\partial}{\partial v} \hat{f}(\mathbf{u}) \boldsymbol{j} .
$$

Because of non-commutativity $\boldsymbol{j}$ appears to the right of $\hat{f}$. Induction over $m, n \in \mathbb{N}$ completes the proof.

Theorem 2.5 (Powers of $\boldsymbol{i}, \boldsymbol{j})$. The QFT of a quaternion module function $\boldsymbol{i}^{m} f(\mathbf{x}) \boldsymbol{j}^{n}$ $\in L^{2}\left(\mathbb{R}^{2} ; \mathbb{H}\right), f \in L^{2}\left(\mathbb{R}^{2} ; \mathbb{H}\right), m, n \in \mathbb{N}_{0}$ is given by

$$
\widehat{\boldsymbol{i}^{m} f \boldsymbol{j}^{n}}(\mathbf{u})=\boldsymbol{i}^{m} \hat{f}(\mathbf{u}) \boldsymbol{j}^{n} .
$$

Proof. Similar to the left and right linearities of table1 1 theorem 2.5 follows directly from the definition 2.1 of the QFT, using the commutation relationships

$$
\exp (-\boldsymbol{i} x u) \boldsymbol{i}^{m}=\boldsymbol{i}^{m} \exp (-\boldsymbol{i} x u) \quad \text { and } \quad \exp (-\boldsymbol{j} y v) \boldsymbol{j}^{n}=\boldsymbol{j}^{n} \exp (-\boldsymbol{j} y v) .
$$

For every $f \in L^{2}\left(\mathbb{R}^{2} ; \mathbb{H}\right)$ we can always rewrite $f=f_{r}+f_{i} \boldsymbol{i}+f_{j} \boldsymbol{j}+f_{k} \boldsymbol{k}$ as in (18) to the form

$$
f=f_{r}+\boldsymbol{i} f_{i}+f_{j} \boldsymbol{j}+\boldsymbol{i} f_{k} \boldsymbol{j}
$$

Accordingly we now can make the following two important observations:

- Theorem 2.5 reduces the computation of the QFT of any $f \in L^{2}\left(\mathbb{R}^{2} ; \mathbb{H}\right)$ to the computation of four QFTs of the real functions $f_{r}, f_{i}, f_{j}, f_{k} \in L^{2}\left(\mathbb{R}^{2} ; \mathbb{R}\right)$ as in

$$
\hat{f}=\hat{f}_{r}+\boldsymbol{i} \hat{f}_{i}+\hat{f}_{j} \boldsymbol{j}+\boldsymbol{i} \hat{f}_{k} \boldsymbol{j} .
$$

- On the other hand theorem 2.5 reveals that every theorem for the QFT of real functions $g \in L^{2}\left(\mathbb{R}^{2} ; \mathbb{R}\right)$ immediately results via (31) in a corresponding theorem for quaternion module functions $f \in L^{2}\left(\mathbb{R}^{2} ; \mathbb{H}\right)$. We simply need to apply the theorem for the QFT of real functions to each of the four real component functions $f_{r}, f_{i}, f_{j}, f_{k} \in L^{2}\left(\mathbb{R}^{2} ; \mathbb{R}\right)$. This fact is rather useful, because often in image processing theorems are only established for real image signals [12. 


\subsection{Example: $G L\left(\mathbb{R}^{2}\right)$ transformation properties of the QFT}

To give an example for the second observation at the end of section 2.2 we use it to generalize the general linear real non-singular transformation property of the QFT of real $2 \mathrm{D}$ functions $f \in L^{2}\left(\mathbb{R}^{2} ; \mathbb{R}\right)$ of $[12$ to quaternion module functions $f \in L^{2}\left(\mathbb{R}^{2} ; \mathbb{H}\right)$.

This property of real $2 \mathrm{D}$ signals states that for

$$
\mathbf{x}^{\prime}=\mathcal{A} \mathbf{x}=(a x+b y) \mathbf{e}_{1}+(c x+d y) \mathbf{e}_{2}
$$

with non-singular real transformation matrix

$$
A=\left(\begin{array}{ll}
a & b \\
c & d
\end{array}\right)
$$

the QFT of a real signal $f: \mathbb{R}^{2} \rightarrow \mathbb{R}$ is 7

$$
\widehat{f(\mathcal{A x})}(\mathbf{u})=\frac{|\operatorname{det} \mathcal{B}|}{2}\left(\hat{f}\left(\mathcal{B}_{+} \mathbf{u}\right)+\hat{f}\left(\mathcal{B}_{-} \mathbf{u}\right)+\boldsymbol{i}\left\{\hat{f}\left(\mathcal{B}_{+} \mathbf{u}\right)-\hat{f}\left(\mathcal{B}_{-} \mathbf{u}\right)\right\} \boldsymbol{j}\right) .
$$

In (34) the two linear real non-singular transformations $\mathcal{B}_{+}$and $\mathcal{B}_{-}$have corresponding matrices and the (same) determinant

$$
\begin{aligned}
& B_{+}=A^{-1^{T}}, \quad B_{-}=\frac{1}{\operatorname{det} A}\left(\begin{array}{cc}
d & c \\
b & a
\end{array}\right), \\
& \operatorname{det} \mathcal{B}=\operatorname{det} B_{+}=\operatorname{det} B_{-}=(\operatorname{det} A)^{-1} .
\end{aligned}
$$

We can now establish the generalization from $f \in L^{2}\left(\mathbb{R}^{2} ; \mathbb{R}\right)$ to $f \in L^{2}\left(\mathbb{R}^{2} ; \mathbb{H}\right)$ functions.

Theorem 2.6. The QFT of a quaternion-module function $f \in L^{2}\left(\mathbb{R}^{2} ; \mathbb{H}\right)$ with a $G L\left(\mathbb{R}^{2}\right)$ transformation $\mathcal{A}$ of its vector arguments (32) is also given by (34).

Proof. We only sketch the proof, because writing out all expressions explicitly would consume too much space:

- Applying (34) and (35) to each component of (31) and

- rearranging the sum (of 16 terms) yields the validity of (34) together with (35) also for quaternion-valued $f \in L^{2}\left(\mathbb{R}^{2} ; \mathbb{H}\right)$.

- It is again crucial that in each term all factors $\boldsymbol{i}$ are always kept to the left and all factors $\boldsymbol{j}$ are always kept to the right.

We remark that resorting to matrices and matrix manipulations is geometrically not very intuitive, so in section 4 an alternative more geometric approach is taken to derive the transformation properties of general $f \in L^{2}\left(\mathbb{R}^{2} ; \mathbb{H}\right)$. This geometric approach has far reaching consequences for the generalization of the QFT, exploited in later sections.

\footnotetext{
${ }^{7}$ Bülow [12] omits the absolute value signs for the determinant of the transformation.
} 
But before geometrically reanalyzing QFT transformation properties we look at the following variant of the QFT with some desirable properties not valid for the QFT of definition 2.1.

\section{The right side quaternion Fourier transform (QFTr)}

We observe that it is not possible to establish a general Plancherel theorem for the QFT of the inner product $(f, g)$ of (11), because the product (11) lacks the cyclic symmetry (10) applied in the proof of theorem 2.2. To obtain a Plancherel theorem it is therefore either necessary to modify the symmetry properties of the inner product as in (12) or to modify the QFT itself. In this section we explore the second possibility.

Definition 3.1 (Right side QFT (QFTr)). The right side quaternion Fourier transform $\stackrel{\nabla}{f}: \mathbb{R}^{2} \rightarrow \mathbb{H}$ of $f \in L^{2}\left(\mathbb{R}^{2} ; \mathbb{H}\right), \mathbf{x}=x \mathbf{e}_{1}+y \mathbf{e}_{2} \in \mathbb{R}^{2}$, and $\mathbf{u}=u \mathbf{e}_{1}+v \mathbf{e}_{2} \in \mathbb{R}^{2}$ is defined as

$$
\stackrel{\triangleright}{f}(\mathbf{u})=\int_{\mathbb{R}^{2}} f(\mathbf{x}) e^{-\boldsymbol{i} x u} e^{-\boldsymbol{j} y v} d^{2} \mathbf{x} \quad \text { with } \quad d^{2} \mathbf{x}=d x d y .
$$

The QFTr is known as Clifford Fourier transform [3, 4, because of the isomorphism $\mathbb{H} \cong C l_{0,2}$. Further freedoms in alternative definitions would be to exchange the order of the exponentials in (36) or to wholly shift both exponential factors to the left side instead. The former would simply exchange the roles of $\boldsymbol{i}$ and $\boldsymbol{j}$, but the latter would not serve our purpose as will soon become clear. The QFTr can be inverted [3, 4] using

$$
f(\mathbf{x})=\frac{1}{(2 \pi)^{2}} \int_{\mathbb{R}^{2}} \stackrel{\triangleright}{f}(\mathbf{u}) e^{j y v} e^{i x u} d^{2} \mathbf{u},
$$

with $d^{2} \mathbf{u}=d u d v$. Attention needs to be paid to the reversed order of the exponential factors in (37) compared to (36).

\subsection{Properties of the QFTr}

For general $f, g \in L^{2}\left(\mathbb{R}^{2} ; \mathbb{H}\right)$ left linearity and dilation properties of table 1 hold. The left linearity coefficients can now be fully quaternionic constants $\alpha^{\prime}, \beta^{\prime} \in \mathbb{H}$.

But $\mathbf{x}$-shift, partial derivative, and powers of $x^{m} y^{n}$ properties need to be modified as in table 2 Regarding (11) it is clear that $\boldsymbol{i} f=f \boldsymbol{i}$ holds iff $f=f_{r}+$

${ }^{8}$ The positions of the real scalars $\alpha, \beta$ before or after the functions $f, g$ do not matter.

${ }^{9}$ Only for quaternion module functions $f \in L^{2}\left(\mathbb{R}^{2} ; \mathbb{H}\right)$ with $\boldsymbol{i} f=f \boldsymbol{i}$, i.e. $f=f_{r}+\boldsymbol{i} f_{i}$ with $f_{r}, f_{i} \in$ $L^{2}\left(\mathbb{R}^{2} ; \mathbb{R}\right)$ do we get $\mathcal{F}_{\triangleright}\left\{f\left(\mathbf{x}-\mathbf{x}_{0}\right)\right\}(\mathbf{u})=e^{-i x_{0} u} \stackrel{\triangleright}{f}(\mathbf{u}) e^{-\boldsymbol{j} y_{0} v}$.

${ }^{10}$ Only for $\boldsymbol{i} f=f \boldsymbol{i}$ do we get $\mathcal{F}_{\triangleright}\left\{\frac{\partial^{m+n}}{\partial x^{m} \partial y^{n}} f\right\}(\mathbf{u})=(\boldsymbol{i} u)^{m}{ }^{\triangleright} f(\mathbf{u})(\boldsymbol{j} v)^{n}$.

${ }^{11}$ Only for $\boldsymbol{i} f=f \boldsymbol{i}$ do we get $\mathcal{F}_{\triangleright}\left\{x^{m} y^{n} f\right\}(\mathbf{u})=\boldsymbol{i}^{m} \frac{\partial^{m+n}}{\partial u^{m} \partial v^{n}} \stackrel{\triangleright}{ } f(\mathbf{u}) \boldsymbol{j}^{n}$.

${ }^{12}$ Here the powers of $\boldsymbol{i}, \boldsymbol{j}$ law is a direct consequence of the left linearity.

${ }^{13}$ Compare theorem 3.2

${ }^{14} \mathrm{~A}$ direct consequence of symmetrizing theorem 3.2 
TABLE 2. Properties of the right sided quaternion Fourier transform (QFTr) of quaternion functions (Quat. Funct.) $f, g \in$ $L^{2}\left(\mathbb{R}^{2} ; \mathbb{H}\right)$, with $\mathbf{x}, \mathbf{u} \in \mathbb{R}^{2}$, constants $\alpha, \beta \in \mathbb{R}, \alpha^{\prime}, \beta^{\prime} \in \mathbb{H}$, $a, b \in \mathbb{R} \backslash\{0\}, \mathbf{x}_{0}=x_{0} \mathbf{e}_{1}+y_{0} \mathbf{e}_{2}, \mathbf{u}_{0}=u_{0} \mathbf{e}_{1}+v_{0} \mathbf{e}_{2} \in \mathbb{R}^{2}$ and $m, n \in \mathbb{N}$.

\begin{tabular}{|c|c|c|}
\hline Property & Quat. Funct. & QFTr \\
\hline Linearity $^{8}$ & $\alpha f(\mathbf{x})+\beta g(\mathbf{x})$ & $\alpha f(\mathbf{u})+\beta^{\triangleright} g(\mathbf{u})$ \\
\hline $\begin{array}{l}\text { Left linearity } \\
\text { x-Shift } 9\end{array}$ & $\begin{array}{l}\alpha^{\prime} f(\mathbf{x})+\beta^{\prime} g(\mathbf{x}) \\
f\left(\mathbf{x}-\mathbf{x}_{0}\right)\end{array}$ & $\begin{array}{l}\alpha^{\prime} f(\mathbf{u})+\beta^{\prime \triangleright} g(\mathbf{u}) \\
\mathcal{F}_{\triangleright}\left\{f e^{-\boldsymbol{i} x_{0} u}\right\}(\mathbf{u}) e^{-\boldsymbol{j} y_{0} v}\end{array}$ \\
\hline Dilation & $f\left(a x \mathbf{e}_{1}+b y \mathbf{e}_{2}\right)$ & $\frac{1}{|a b|} f\left(\frac{u}{a} \mathbf{e}_{1}+\frac{v}{b} \mathbf{e}_{2}\right)$ \\
\hline Part. deriv 10 & $\frac{\partial^{m+n}}{\partial x^{m} \partial y^{n}} f(\mathbf{x}) i^{-m}$ & $u^{m} f(\mathbf{u})(\boldsymbol{j} v)^{n}$ \\
\hline Powers ${ }^{11}$ of $x, y$ & $x^{m} y^{n} f(\mathbf{x}) \boldsymbol{i}^{-m}$ & $\frac{\partial^{m+n}}{\partial u^{m} \partial v^{n}} f(\mathbf{u}) \boldsymbol{j}^{n}$ \\
\hline Powers ${ }^{12}$ of $\boldsymbol{i}, \boldsymbol{j}$ & $\boldsymbol{i}^{m} \boldsymbol{j}^{n} f(\mathbf{x})$ & $\boldsymbol{i}^{m} \boldsymbol{j}^{n} f(\mathbf{u})$ \\
\hline Plancherel $\underline{13}$ & $(f, g)=$ & $\frac{1}{(2 \pi)^{2}}(\stackrel{\triangleright}{f}, \stackrel{\triangleright}{g})$ \\
\hline Plancherel $\underline{14}^{14}$ & $\langle f, g\rangle=$ & $\frac{1}{(2 \pi)^{2}}\langle\stackrel{\triangleright}{f}, \stackrel{\vec{g}}{g}\rangle$ \\
\hline Parseval & $\|f\|=$ & $\frac{1}{2 \pi}\|f\|$ \\
\hline
\end{tabular}

$f_{i} \boldsymbol{i}, f_{r}, f_{i} \in \mathbb{R}$, which is slightly more general than the restriction of [12] to $f=$ $f_{r} \in \mathbb{R}$. A modulation property analogous to the one in table 1 does not hold. It is obstructed by the non-commutativity of the exponential factors

$$
\exp \left(\boldsymbol{j} y v_{0}\right) \exp (\boldsymbol{i} x u) \neq \exp (\boldsymbol{i} x u) \exp \left(\boldsymbol{j} y v_{0}\right) .
$$

For a powers of $\boldsymbol{i}, \boldsymbol{j}$ property to hold for the QFTr, we need to shift the factors $\boldsymbol{j}^{n}$ also to the left of the quaternion function $f(\mathbf{x})$.

For fully general quaternion-valued $f, g \in L^{2}\left(\mathbb{R}^{2} ; \mathbb{H}\right)$ we can establish for the QFTr the following quaternion-valued Plancherel theorem based on the inner product (11).

Theorem 3.2 (QFTr Plancherel). The (quaternion-valued) inner product (11) of two quaternion module functions $f, g \in L^{2}\left(\mathbb{R}^{2} ; \mathbb{H}\right)$ is given by the inner product of the corresponding QFTrs $\stackrel{\triangleright}{f}$ and $\stackrel{\triangleright}{g}$

$$
(f, g)=\frac{1}{(2 \pi)^{2}}(\stackrel{\triangleright}{f}, \stackrel{\triangleright}{g}) .
$$


Proof. For $f, g \in L^{2}\left(\mathbb{R}^{2} ; \mathbb{H}\right)$ we calculate the inner product (11)

$$
\begin{gathered}
(f, g)=\int_{\mathbb{R}^{2}} f(\mathbf{x}) \tilde{g}(\mathbf{x}) d^{2} \mathbf{x} \\
=\frac{1}{(2 \pi)^{2}} \int_{\mathbb{R}^{2}} \int_{\mathbb{R}^{2}} f(\mathbf{u}) e^{\boldsymbol{j} v y} e^{\boldsymbol{i} u x} d^{2} \mathbf{u} \tilde{g}(\mathbf{x}) d^{2} \mathbf{x} \\
=\frac{1}{(2 \pi)^{2}} \int_{\mathbb{R}^{2}} f(\mathbf{u}) \int_{\mathbb{R}^{2}} e^{\boldsymbol{j} v y} e^{\boldsymbol{i} u x} \tilde{g}(\mathbf{x}) d^{2} \mathbf{x} d^{2} \mathbf{u} \\
=\frac{1}{(2 \pi)^{2}} \int_{\mathbb{R}^{2}} f(\mathbf{u})\left[\int_{\mathbb{R}^{2}} g(\mathbf{x}) e^{-\boldsymbol{i} u x} e^{-\boldsymbol{j} v y} d^{2} \mathbf{x}\right]^{\sim} d^{2} \mathbf{u} \\
=\frac{1}{(2 \pi)^{2}} \int_{\mathbb{R}^{2}}^{\triangleright} f(\mathbf{u}) \stackrel{\tilde{\triangleright}}{g}(\mathbf{u}) d^{2} \mathbf{u}=\frac{1}{(2 \pi)^{2}}(\stackrel{\triangleright}{f}, \stackrel{\triangleright}{g}) .
\end{gathered}
$$

In the second equality of (40) we replaced $f$ with its inverse QFTr expression (37). In the third equality we exchanged the order of integration. For the fourth equality we simply pulled the reversion outside the square brackets [...] and obtained the QFTr $\stackrel{\triangleright}{g}(\mathbf{u})$, which proves (39) according to (11).

For $g=f$ theorem 3.2 has a corresponding QFTr Parseval theorem as a direct corollary.

Corollary 3.3 (QFTr Parseval). The $L^{2}\left(\mathbb{R}^{2} ; \mathbb{H}\right)$-norm of a quaternion module function $f \in L^{2}\left(\mathbb{R}^{2} ; \mathbb{H}\right)$ is given by the $L^{2}\left(\mathbb{R}^{2} ; \mathbb{H}\right)$-norm of its QFTr $\stackrel{\triangleright}{f}$ multiplied by $1 /(2 \pi)$

$$
\|f\|=\frac{1}{2 \pi}\|\stackrel{f}{\triangleright}\|=\frac{1}{2 \pi}\|\hat{f}\| .
$$

Proof. The first identity follows from setting $g=f$ in theorem 3.2(QFTr Plancherel). The second identity follows from comparing with corollary 2.3 (QFT Parseval).

To facilitate the use of the QFTr and comparison with the QFT (table1) we list the main QFTr properties in table 2.

\section{Understanding the $G L\left(\mathbb{R}^{2}\right)$ transformation properties of the QFT}

We begin with noting that the matrix transformation law (34), derived by Bülow 12. for real signals $f \in L^{2}\left(\mathbb{R}^{2} ; \mathbb{R}\right)$, and generalized in theorem 2.6 of section 2.3 to quaternion-valued signals $15 f \in L^{2}\left(\mathbb{R}^{2} ; \mathbb{H}\right)$, with four terms on the right side, allows no straightforward geometric interpretation. Yet a clear geometric interpretation is not only needed in many applications, such an interpretation is also very instructive in order to successfully generalize the QFT to higher dimensions.

\footnotetext{
${ }^{15}$ Remember that Bülow [12 proved his transformation law only for real signals. But in theorem 2.6 of section 2.3 , we used (31) and theorem 2.5 to generalize from real signals $f \in L^{2}\left(\mathbb{R}^{2}, \mathbb{R}\right)$ to quaternion valued signals $f \in L^{2}\left(\mathbb{R}^{2}, \mathbb{H}\right)$.
} 
Toward this aim we observe, that the split (21) of the exponentials $K$ under the QFT integral results in two (single exponential) complex kernels $K_{ \pm}$with complex units $\boldsymbol{i}$ (or $\boldsymbol{j}$ ) apart from the right (or left) factor $(1 \pm \boldsymbol{k}) / 2$.

This and the known elegant monomial transformation properties of complex Fourier transforms (also preserved in the Clifford FT of [9]) motivates us to geometrically re-analyze the $G L\left(\mathbb{R}^{2}\right)$ transformation properties of the QFT of $f \in L^{2}\left(\mathbb{R}^{2} ; \mathbb{H}\right)$ in terms of its two components $f_{ \pm}$as given in (19).

Theorem 4.1 (QFT of $f_{ \pm}$). The QFT of the $f_{ \pm}$split parts of a quaternion module function $f \in L^{2}\left(\mathbb{R}^{2}, \mathbb{H}\right)$ have the complex forms

$$
\hat{f}_{ \pm}=\int_{\mathbb{R}^{2}} f_{ \pm} e^{-\boldsymbol{j}(y v \mp x u)} d^{2} x=\int_{\mathbb{R}^{2}} e^{-\boldsymbol{i}(x u \mp y v)} f_{ \pm} d^{2} x
$$

Proof.

$$
\begin{gathered}
\hat{f}_{ \pm}=\int_{\mathbb{R}^{2}} e^{-\boldsymbol{i} x u}\left\{f_{r} \pm f_{k}+\boldsymbol{i}\left(f_{i} \mp f_{j}\right)\right\} \frac{1 \pm \boldsymbol{k}}{2} e^{-\boldsymbol{j} y v} d^{2} x \\
=\int_{\mathbb{R}^{2}}\left\{f_{r} \pm f_{k}+\boldsymbol{i}\left(f_{i} \mp f_{j}\right)\right\} e^{-\boldsymbol{i} x u} \frac{1 \pm \boldsymbol{k}}{2} e^{-\boldsymbol{j} y v} d^{2} x \\
=\int_{\mathbb{R}^{2}}\left\{f_{r} \pm f_{k}+\boldsymbol{i}\left(f_{i} \mp f_{j}\right)\right\} \underbrace{\frac{1 \pm \boldsymbol{k}}{2} e^{-\boldsymbol{j}(y v \mp x u)}}_{=K_{ \pm}} d^{2} x \\
\stackrel{\underline{20}}{=} \int_{\mathbb{R}^{2}} f_{ \pm} e^{-\boldsymbol{j}(y v \mp x u)} d^{2} x=\int_{\mathbb{R}^{2}} e^{-\boldsymbol{i}(x u \mp y v)} f_{ \pm} d^{2} x,
\end{gathered}
$$

where for the third equality we did a number of quaternion algebra manipulations, involving Euler's formula and trigonometric addition theorems. The last equality of (43) follows analogously by replacing $f_{ \pm}$with the third expression in (20), etc.

We learn from the third line of (43) that the behavior of the two parts (21) under automorphisms $\mathcal{A} \in G L\left(\mathbb{R}^{2}\right)$ also determines the automorphism properties of the QFTs $\hat{f}_{ \pm}$, where due to theorem 2.5 the QFT operation and the split operation (19) commute.

\subsection{Geometric interpretation and coordinate independent formulation of $G L\left(\mathbb{R}^{2}\right)$ transformations of the QFT}

We begin with noting that according to the polar decomposition theorem [17] every automorphism $\mathcal{A} \in G L\left(\mathbb{R}^{2}\right)$ has a unique decomposition $\mathcal{A}=\mathcal{T} \mathcal{R}=\mathcal{R} \mathcal{S}$, where $\mathcal{R}$ is a rotation and $\mathcal{T}$ and $\mathcal{S}$ are symmetric with positive and negative eigenvalues.

Positive eigenvalues correspond to stretches by the eigenvalue in the direction of the eigenvector. Negative eigenvalues correspond to reflections at the line (hyperplane) normal to the eigenvector, composed with stretches by the absolute value of the eigenvalue in the direction of the eigenvector.

Stretches (positive eigenvalues) $\mathcal{D} \in G L\left(\mathbb{R}^{2}\right)$ were already fully treated in 12. (compare also table 1). 
Rotations correspond to two reflections [18, 19] at lines subtending half the angle of the resulting rotation $\mathcal{R}_{\mathrm{ab}}=\mathcal{U}_{\mathrm{a}} \mathcal{U}_{\mathrm{b}}$. The elementary transformations that compose all automorphisms $\mathcal{A} \in G L\left(\mathbb{R}^{2}\right)$ are therefore stretches and reflections.

In geometric algebra reflections $\mathcal{U}_{\mathbf{n}}$ at a hyperplane (line in 2D) through the origin can be characterized by normal vectors $\mathbf{n}$

$$
\mathcal{U}_{\mathbf{n}} \mathbf{x}=-\mathbf{n}^{-1} \mathbf{x n}
$$

The length of $\mathbf{n}$ does not matter. $\mathcal{U}_{\mathbf{n}}$ preserves (reverses) the component parallel (perpendicular) to the hyperplane of reflection.

With the vectors $\mathbf{x}=x \mathbf{e}_{1}+y \mathbf{e}_{2}, \mathbf{u}=u \mathbf{e}_{1}+v \mathbf{e}_{2}$ we now rewrite coordinate free 16 the angles in the exponentials of $\hat{f}_{ \pm}$as

$$
-x u+y v=\mathbf{x} \cdot\left(\mathcal{U}_{\mathbf{e}_{1}} \mathbf{u}\right), \quad x u+y v=\mathbf{x} \cdot \mathbf{u} .
$$

Hence we get for the QFTs of $f_{ \pm}$

$$
\hat{f}_{+}=\int_{\mathbb{R}^{2}} f_{+} e^{-j \mathbf{j} \mathbf{x} \cdot\left(\mathcal{U}_{\mathbf{e}_{1}} \mathbf{u}\right)} d^{2} x, \quad \hat{f}_{-}=\int_{\mathbb{R}^{2}} f_{-} e^{-j \mathbf{x} \cdot \mathbf{u}} d^{2} x .
$$

The QFT of $f_{-}$is analogous to a complex 2D Fourier transform, only in general $f_{-}$and the exponential factor do not commute. The QFT of $f_{+}$is similar except for the reflection $\mathcal{U}_{\mathbf{e}_{1}}$.

We are now in a position to apply any automorphism $\mathcal{A} \in G L\left(\mathbb{R}^{2}\right)$ to the spatial argument of the $f_{ \pm}$components of any $f \in L^{2}\left(\mathbb{R}^{2}, \mathbb{H}\right)$. We begin with

$$
\begin{gathered}
\left.\widehat{f_{-}(\mathcal{A} \mathbf{x}}\right)(\mathbf{u})=\int_{\mathbb{R}^{2}} f_{-}(\mathcal{A} \mathbf{x}) e^{-\boldsymbol{j} \mathbf{x} \cdot \mathbf{u}} d^{2} x \\
\stackrel{\mathbf{z}=\mathcal{A} \mathbf{x}}{=} \int_{\mathbb{R}^{2}} f_{-}(\mathbf{z}) e^{-\boldsymbol{j}\left(\mathcal{A}^{-1} \mathbf{z}\right) \cdot \mathbf{u}}\left|\operatorname{det} \mathcal{A}^{-1}\right| d^{2} z \\
=\left|\operatorname{det} \mathcal{A}^{-1}\right| \int_{\mathbb{R}^{2}} f_{-}(\mathbf{z}) e^{-\boldsymbol{j} \mathbf{z} \cdot\left(\overline{\mathcal{A}^{-1}} \mathbf{u}\right)} d^{2} z \\
=\left|\operatorname{det} \mathcal{A}^{-1}\right| \hat{f}_{-}\left(\overline{\mathcal{A}^{-1}} \mathbf{u}\right),
\end{gathered}
$$

where $\overline{\mathcal{A}^{-1}}$ indicates the adjoint automorphism of $\mathcal{A}^{-1}$. The absolute value of the determinant $\operatorname{det} \mathcal{A}^{-1}$ needs to be used, because of the interchange of integration

\footnotetext{
${ }^{16}$ The fact that the reflection $\mathcal{U}_{\mathbf{e}_{1}}$ with the special hyperplane normal to vector $\mathbf{e}_{1}$ is needed stems from the arbitrary initial association of the $\mathbf{e}_{1}$-coordinate product $x u$ with $\boldsymbol{i}$ and of the $\mathbf{e}_{2}$-coordinate product $y v$ with $\boldsymbol{j}$.
} 
boundaries for a negative determinant. We continue with

$$
\begin{gathered}
\left.\widehat{f_{+}(\mathcal{A} \mathbf{x}}\right)(\mathbf{u})=\int_{\mathbb{R}^{2}} f_{+}(\mathcal{A} \mathbf{x}) e^{-j \mathbf{x} \cdot\left(\mathcal{U}_{\mathbf{e}_{1}} \mathbf{u}\right)} d^{2} x \\
\stackrel{\mathbf{z}=\mathcal{A} \mathbf{x}}{=} \int_{\mathbb{R}^{2}} f_{+}(\mathbf{z}) e^{-\boldsymbol{j}\left(\mathcal{A}^{-1} \mathbf{z}\right) \cdot\left(\mathcal{U}_{\mathbf{e}_{1}} \mathbf{u}\right)}\left|\operatorname{det} \mathcal{A}^{-1}\right| d^{2} z \\
=\left|\operatorname{det} \mathcal{A}^{-1}\right| \int_{\mathbb{R}^{2}} f_{+}(\mathbf{z}) e^{\left.-\boldsymbol{j} \mathbf{z} \cdot \overline{\mathcal{A}^{-1}} \mathcal{U}_{\mathbf{e}_{1}} \mathbf{u}\right)} d^{2} z \\
=\left|\operatorname{det} \mathcal{A}^{-1}\right| \int_{\mathbb{R}^{2}} f_{+}(\mathbf{z}) e^{-\boldsymbol{j} \mathbf{z} \cdot\left(\mathcal{U}_{\mathbf{e}_{1}} \mathcal{U}_{\mathbf{e}_{1}} \overline{\mathcal{A}^{-1}} \mathcal{U}_{\mathbf{e}_{1}} \mathbf{u}\right)} d^{2} z \\
=\left|\operatorname{det} \mathcal{A}^{-1}\right| \hat{f}_{+}\left(\mathcal{U}_{\mathbf{e}_{1}} \overline{\mathcal{A}^{-1}} \mathcal{U}_{\mathbf{e}_{1}} \mathbf{u}\right),
\end{gathered}
$$

which is very similar to the previous calculation for $\hat{f}_{-}$. The only difference is that in line 4 we insert $1=\mathcal{U}_{\mathbf{e}_{1}} \mathcal{U}_{\mathbf{e}_{1}}$ before $\overline{\mathcal{A}^{-1}}$, and that the argument of the transformed $\hat{f}_{+}$now has the reflected version $\mathcal{U}_{\mathbf{e}_{1}} \overline{\mathcal{A}^{-1}} \mathcal{U}_{\mathbf{e}_{1}}$ of the adjoint inverse transformation $\overline{\mathcal{A}^{-1}}$. Recombining $\hat{f}_{+}$and $\hat{f}_{-}$we get from (47) and (48)

Theorem 4.2 $\left(G L\left(\mathbb{R}^{2}\right)\right.$ transformation properties of the QFT). The $Q F T$ of a quaternion module function $f \in L^{2}\left(\mathbb{R}^{2} ; \mathbb{H}\right)$ with a $G L\left(\mathbb{R}^{2}\right)$ transformation $\mathcal{A}$ of its vector argument is given by

$$
\widehat{f(\mathcal{A x})}(\mathbf{u})=\left|\operatorname{det} \mathcal{A}^{-1}\right|\left\{\hat{f}_{-}\left(\overline{\mathcal{A}^{-1}} \mathbf{u}\right)+\hat{f}_{+}\left(\mathcal{U}_{\mathbf{e}_{1}} \overline{\mathcal{A}^{-1}} \mathcal{U}_{\mathbf{e}_{1}} \mathbf{u}\right)\right\}
$$

Theorem 4.2 corresponds exactly to equation (34) with (35), if the matrix expression (33) is used for the automorphism $\mathcal{A}$ and if the $f_{ \pm}$split formulas (19) are used. The four terms of (34) together with all the matrices involved therefore get in theorem 4.2 a clear geometric interpretation. In order to be even more explicit we specify below the full geometric algebra expressions for stretches, reflections and rotations.

\subsection{Explicit examples: stretches, reflections \& rotations}

To deepen our geometrical understanding we now look at stretches, reflections (and rotations) which compose every general automorphism $\mathcal{A} \in G L\left(\mathbb{R}^{2}\right)$.

Stretches expressed by $\mathcal{A}_{s} \mathbf{x}=$ ax $\mathbf{e}_{1}+$ by $\mathbf{e}_{2}$, with $a, b \in \mathbb{R} \backslash\{0\}$, result because of $\mathcal{U}_{\mathbf{e}_{1}} \mathcal{A}_{s} \mathcal{U}_{\mathbf{e}_{1}}=\mathcal{A}_{s}$ in

$$
\left.\widehat{f\left(\mathcal{A}_{s} \mathbf{x}\right.}\right)(\mathbf{u})=\left|\operatorname{det} \mathcal{A}_{s}^{-1}\right| \hat{f}\left(\mathcal{A}_{s}^{-1} \mathbf{u}\right)=\frac{1}{|a b|} \hat{f}\left(\frac{u}{a} \mathbf{e}_{1}+\frac{v}{b} \mathbf{e}_{2}\right) .
$$

Reflections in hyperplanes normal to a expressed by $\mathcal{U}_{\mathbf{a}} \mathbf{x}=-\mathbf{a}^{-1} \mathbf{x a}$, with $\left|\operatorname{det} \mathcal{U}_{\mathbf{a}}\right|=1, \overline{\mathcal{U}_{\mathbf{a}}}=\mathcal{U}_{\mathbf{a}}, \mathcal{U}_{\mathbf{e}_{1}} \mathcal{U}_{\mathbf{a}} \mathcal{U}_{\mathbf{e}_{1}}=\mathcal{U}_{\mathbf{a}^{\prime}}$, and $\mathbf{a}^{\prime}=\mathcal{U}_{\mathbf{e}_{1}} \mathbf{a}$ result in

$$
\left.\widehat{f\left(\mathcal{U}_{\mathbf{a}} \mathbf{x}\right.}\right)(\mathbf{u})=\hat{f}_{-}\left(\mathcal{U}_{\mathbf{a}} \mathbf{u}\right)+\hat{f}_{+}\left(\mathcal{U}_{\mathbf{a}^{\prime}} \mathbf{u}\right) .
$$

Finally rotations (equivalent to two reflections at lines subtending half the rotation angle) expressed by $\mathcal{R}_{\mathbf{a b}} \mathbf{x}=\mathcal{U}_{\mathrm{b}} \mathcal{U}_{\mathbf{a}} \mathbf{x}$, with $\left|\operatorname{det} \mathcal{R}_{\mathbf{a b}}\right|=1, \mathcal{R}_{\mathrm{ab}}^{-1}=\mathcal{R}_{\mathrm{ba}}$, 
and $\mathcal{U}_{\mathbf{a}^{\prime}} \mathcal{U}_{\mathbf{b}^{\prime}}=\mathcal{U}_{\mathbf{e}_{1}} \mathcal{R}_{\mathbf{a b}}^{-1} \mathcal{U}_{\mathbf{e}_{1}}$, result in

$$
\begin{aligned}
\left.f \widehat{\left(\mathcal{R}_{\mathbf{a b}} \mathbf{x}\right.}\right)(\mathbf{u}) & =\hat{f}\left(\mathcal{U}_{\mathbf{b}} \mathcal{U}_{\mathbf{a}} \mathbf{x}\right)(\mathbf{u})=\hat{f}_{-}\left(\mathcal{U}_{\mathbf{a}} \mathcal{U}_{\mathbf{b}} \mathbf{u}\right)+\hat{f}_{+}\left(\mathcal{U}_{\mathbf{a}^{\prime}} \mathcal{U}_{\mathbf{b}^{\prime}} \mathbf{u}\right) \\
& =\hat{f}_{-}\left(\mathcal{R}_{\mathbf{a b}}^{-1} \mathbf{u}\right)+\hat{f}_{+}\left(\mathcal{U}_{\mathbf{e}_{1}} \mathcal{R}_{\mathbf{a b}}^{-1} \mathcal{U}_{\mathbf{e}_{1}} \mathbf{u}\right)
\end{aligned}
$$

In two dimensions 17 the formula for rotations of the spatial argument of a quaternion module function $f$ subject to the QFT can be further simplified to

$$
\left.f \widehat{\left(\mathcal{R}_{\mathbf{a b}} \mathbf{x}\right.}\right)(\mathbf{u}) \stackrel{\text { in } 2 \mathrm{D}}{=} \hat{f}_{-}\left(\mathcal{R}_{\mathbf{a b}}^{-1} \mathbf{u}\right)+\hat{f}_{+}\left(\mathcal{R}_{\mathbf{a b}} \mathbf{u}\right)
$$

because in two dimensions we have $\mathcal{U}_{\mathbf{e}_{1}} \mathcal{R}_{\mathrm{ab}}^{-1} \mathcal{U}_{\mathbf{e}_{1}}=\mathcal{R}_{\mathrm{ab}}$.

Theorems 4.1 and 4.2 together with their clear geometric interpretation with the help of geometric algebra pave the way for wide-ranging generalizations of the QFT of definition 2.1. In this paper we cannot fully treat all possible generalizations. But in order to demonstrate the method, we show in the following section how to generalize the QFT to a new general non-commutative Fourier transformation of functions from spacetime $\mathbb{R}^{3,1}$ to the spacetime algebra 20 of $\mathbb{R}^{3,1}$, i.e. to the Clifford geometric algebra $C l_{3,1}$. An intermediate step will be the generalization to a new Fourier transform of functions from spacetime $\mathbb{R}^{3,1}$ to a volume-time subalgebra of the spacetime algebra.

\section{Generalization of the QFT to a new spacetime algebra Fourier transform}

We begin by recalling quaternion algebra to Clifford subalgebra isomorphisms such as $\mathbb{H} \cong C l(0,2) \cong C l^{+}(3,0)$. Such isomorphisms together with the generalized $G L\left(\mathbb{R}^{n, m}\right)$ transformation laws for $\left\{\hat{f}_{ \pm}(\mathcal{A x})\right\}(\mathbf{u})$ allow us now to generalize the QFT to higher dimensions.

This indeed opens up a vast new field of related multivector Fourier transforms, which are in general non-commutative.

\subsection{QFT generalization to volume-time functions}

One of these quaternion algebra to Clifford sub-algebra isomorphisms that is of particular relevance in physics exists with a subalgebra of the spacetime algebra $C l_{3,1}$. We express this isomorphism by introducing an orthonormal (grade 1) vector basis for $\mathbb{R}^{3,1}$

$$
\left\{\mathbf{e}_{0}, \mathbf{e}_{1}, \mathbf{e}_{2}, \mathbf{e}_{3}\right\}, \quad-\mathbf{e}_{0}^{2}=\mathbf{e}_{1}^{2}=\mathbf{e}_{2}^{2}=\mathbf{e}_{3}^{2}=1
$$

Using this vector basis of $\mathbb{R}^{3,1}$, the spatial unit volume trivector $i_{3}$ and total fourdimensional (hyper volume) pseudoscalar $i_{4}$ can be expressed by

$$
i_{3}=\mathbf{e}_{1} \mathbf{e}_{2} \mathbf{e}_{3}, \quad i_{3}^{2}=-1, \quad i_{4}=\mathbf{e}_{0} \mathbf{e}_{1} \mathbf{e}_{2} \mathbf{e}_{3}, \quad i_{4}^{2}=-1 .
$$

We emphasize the fact that the vector $\mathbf{e}_{0}$, the $3 \mathrm{D}$ volume trivector $i_{3}$, and the $4 \mathrm{D}$ pseudoscalar $i_{4}$, all square to minus one. Examining the geometric algebra

\footnotetext{
${ }^{17}$ In section 5 we generalize theorem 4.2 to higher dimensions, but for rotations the expression
} for $\hat{f}_{+}$on the right hand side of (53) will in general not be valid for higher dimensions. 
multiplication laws of $\mathbf{e}_{0}, i_{3}$, and $i_{4}$, shows indeed that the arising subalgebra $V_{t}$ of the spacetime algebra is isomorphic (see sections 4.1 and 4.2 of [21]) to the quaternion algebra $\mathbb{H}$

$$
V_{t} \cong \mathbb{H},
$$

where we use $V_{t}$ to denote the volume-time subalgebra of $C l_{3,1}$ with subalgebra basis

$$
\left\{1, \mathbf{e}_{0}, i_{3}, i_{4}\right\}
$$

Note especially that

$$
i_{3}=\mathbf{e}_{0}\left(-i_{4}\right)=\mathbf{e}_{0} i_{4}^{-1}=\mathbf{e}_{0}^{*},
$$

which shows that $i_{3}$ is dual to $\mathbf{e}_{0}$ in $C l_{3,1}$.

Based on the isomorphism (56) we now define a Fourier transform for volumetime module functions $f \in L^{2}\left(\mathbb{R}^{3,1} ; V_{t}\right)$.

Definition 5.1 (Volume-time Fourier transform (VtFT)). The volume-time Fourier transform $\stackrel{\circ}{f}: \mathbb{R}^{3,1} \rightarrow V_{t}$ of volume-time module functions $f \in L^{2}\left(\mathbb{R}^{3,1} ; V_{t}\right)$, with spacetime vectors $\mathbf{x}=t \mathbf{e}_{0}+\vec{x} \in \mathbb{R}^{3,1}, \vec{x}=x \mathbf{e}_{1}+y \mathbf{e}_{2}+z \mathbf{e}_{3} \in \mathbb{R}^{3}$, and spacetime frequency vectors $\mathbf{u}=s \mathbf{e}_{0}+\vec{u} \in \mathbb{R}^{3,1}, \vec{u}=u \mathbf{e}_{1}+v \mathbf{e}_{2}+w \mathbf{e}_{3} \in \mathbb{R}^{3}$ is defined as

$$
\stackrel{\circ}{f}(\mathbf{u})=\int_{\mathbb{R}^{3,1}} e^{-\mathbf{e}_{0} t s} f(\mathbf{x}) e^{-i_{3} \vec{x} \cdot \vec{u}} d^{4} \mathbf{x},
$$

with the differential spacetime integration volume $d^{4} \mathbf{x}=d t d x d y d z$.

The VtFT can be inverted in close analogy to (17) by using

$$
f(\mathbf{x})=\frac{1}{(2 \pi)^{4}} \int_{\mathbb{R}^{3,1}} e^{\mathbf{e}_{0} t s} \stackrel{\circ}{f}(\mathbf{u}) e^{i_{3} \vec{x} \cdot \vec{u}} d^{4} \mathbf{u},
$$

with $d^{4} \mathbf{u}=d s d u d v d w$.

The $f_{ \pm}$split (19) combined with the isomorphism (56) now yields for volumetime module functions $f \in L^{2}\left(\mathbb{R}^{3,1} ; V_{t}\right)$

$$
f=f_{+}+f_{-}, \quad f_{+}=\frac{1}{2}\left(f+\mathbf{e}_{0} f i_{3}\right), \quad f_{-}=\frac{1}{2}\left(f-\mathbf{e}_{0} f i_{3}\right) .
$$

Rewriting the split (61) with the duality relation (58) to

$$
f_{ \pm}=\frac{1}{2}\left(f \pm \mathbf{e}_{0} f \mathbf{e}_{0}^{*}\right)
$$

shows that it naturally only depends on the physical spacetime split, i.e. on the choice of the time direction $\mathbf{e}_{0}$. Applying our new VtFT of definition 5.1 to the split functions $f_{ \pm}$of (62) results in a VtFT formula which corresponds to theorem 4.1

$$
\stackrel{\circ}{f_{ \pm}}=\int_{\mathbb{R}^{3,1}} f_{ \pm} e^{-i_{3}(\vec{x} \cdot \vec{u} \mp t s)} d^{4} x=\int_{\mathbb{R}^{3,1}} e^{-\mathbf{e}_{0}(t s \mp \vec{x} \cdot \vec{u})} f_{ \pm} d^{4} x .
$$

Note especially that the $\stackrel{\circ}{f}$, part in (63) has the kernel with the flat Minkowski metric $t s-\vec{x} \cdot \vec{u}$ in the exponent. (Compare section 5.3 for further interpretation.) 
Definition 5.1 preserves the form of the $G L$ transformation properties of section 4. We get the $G L\left(\mathbb{R}^{3,1}\right)$ transformation properties of (59) simply by inserting in theorem 4.2 transformations $\mathcal{A} \in G L\left(\mathbb{R}^{3,1}\right)$ and replacing $\mathcal{U}_{\mathbf{e}_{1}}$ by $\mathcal{U}_{\mathbf{e}_{0}}$.

Theorem 5.2 $\left(G L\left(\mathbb{R}^{3,1}\right)\right.$ transformation properties of the VtFT). The VtFT of a $V_{t}$ module function $f \in L^{2}\left(\mathbb{R}^{2} ; V_{t}\right)$ with a $G L\left(\mathbb{R}^{3,1}\right)$ transformation $\mathcal{A}$ of its vector argument is given by

$$
\{f(\mathcal{A} \mathbf{x})\}^{\circ}(\mathbf{u})=\left|\operatorname{det} \mathcal{A}^{-1}\right|\left\{\stackrel{\circ}{f}\left(\overline{\mathcal{A}^{-1}} \mathbf{u}\right)+\stackrel{\circ}{f_{+}}\left(\mathcal{U}_{\mathbf{e}_{0}} \overline{\mathcal{A}^{-1}} \mathcal{U}_{\mathbf{e}_{0}} \mathbf{u}\right)\right\} .
$$

In physical applications proper Lorentz transformations with $|\operatorname{det} \mathcal{A}|=1$ are most relevant, so the $\left|\operatorname{det} \mathcal{A}^{-1}\right|$ factor in (64) can then naturally be omitted.

For all kinds of applications it is of interest to know whether we can push the QFT generalization established by the VtFT for volume-time module functions $f \in L^{2}\left(\mathbb{R}^{3,1} ; V_{t}\right)$ even further, i.e. if even more general spacetime algebra functions can be treated meaningfully with the VtFT. That this is indeed the case will be shown in the next subsection.

\subsection{Generalization to full spacetime algebra functions}

We now explain how we can drop in the VtFT definition 5.1 the restriction to volume-time functions $f \in L^{2}\left(\mathbb{R}^{3,1} ; V_{t}\right)$. The key to this is found in the commutativity of the unit volume trivector $i_{3}$ of the right side exponential factor in (59) with all spatial vectors $\left\{\mathbf{e}_{1}, \mathbf{e}_{2}, \mathbf{e}_{3}\right\}$

$$
i_{3} \mathbf{e}_{k}=\mathbf{e}_{k} i_{3}, \quad 1 \leq k \leq 3 .
$$

This directly leads us to the right linearity of the VtFT

$$
\begin{gathered}
\{f \alpha\}^{\circ}(\mathbf{u})=\int_{\mathbb{R}^{3,1}} e^{-\mathbf{e}_{0} t s} f(\mathbf{x}) \alpha e^{-i_{3} \vec{x} \cdot \vec{u}} d^{4} \mathbf{x} \\
=\int_{\mathbb{R}^{3,1}} e^{-\mathbf{e}_{0} t s} f(\mathbf{x}) e^{-i_{3} \vec{x} \cdot \vec{u}} d^{4} \mathbf{x} \alpha=\stackrel{\circ}{f}(\mathbf{u}) \alpha, \quad \forall \text { const. } \alpha \in C l_{3,0},
\end{gathered}
$$

where $C l_{3,0}$ is the eight-dimensional Clifford geometric algebra of $\mathbb{R}^{3,0}$, i.e. the $3 \mathrm{D}$ space subalgebra of $C l_{3,1}$ spanned by

$$
\left\{1, \mathbf{e}_{1}, \mathbf{e}_{2}, \mathbf{e}_{3}, \mathbf{e}_{2} \mathbf{e}_{3}, \mathbf{e}_{3} \mathbf{e}_{1}, \mathbf{e}_{1} \mathbf{e}_{2}, i_{3}\right\} .
$$

Naturally this right linearity also holds for the inverse transformation

$$
f(\mathbf{x}) \alpha=\frac{1}{(2 \pi)^{4}} \int_{\mathbb{R}^{3,1}} e^{\mathbf{e}_{0} t s} \stackrel{\circ}{f}(\mathbf{u}) \alpha e^{i_{3} \vec{x} \cdot \vec{u}} d^{4} \mathbf{u} \quad \forall \text { const. } \alpha \in C l_{3,0} .
$$

Now all 16 basis multivectors of $C l_{3,1}$ can be obtained by successive geometric multiplications of 1 and $\mathbf{e}_{0}$ (or alternatively of $i_{3}$ and $i_{4}$, etc.) with the three 
spatial vectors $\left\{\mathbf{e}_{1}, \mathbf{e}_{2}, \mathbf{e}_{3}\right\}$ from the right 18

$$
\begin{gathered}
\left\{1, \mathbf{e}_{1}, \mathbf{e}_{2}, \mathbf{e}_{3}, \mathbf{e}_{2} \mathbf{e}_{3}, \mathbf{e}_{3} \mathbf{e}_{1}, \mathbf{e}_{1} \mathbf{e}_{2}, i_{3},\right. \\
\left.\mathbf{e}_{0}, \mathbf{e}_{0} \mathbf{e}_{1}, \mathbf{e}_{0} \mathbf{e}_{2}, \mathbf{e}_{0} \mathbf{e}_{3}, \mathbf{e}_{0} \mathbf{e}_{2} \mathbf{e}_{3}, \mathbf{e}_{0} \mathbf{e}_{3} \mathbf{e}_{1}, \mathbf{e}_{0} \mathbf{e}_{1} \mathbf{e}_{2}, \mathbf{e}_{0} i_{3}\right\} .
\end{gathered}
$$

We now have laid all the groundwork for the full spacetime algebra generalization of the VtFT of definition 5.1

Definition 5.3 (Spacetime Fourier transform (SFT)). The SFT $\stackrel{\ominus}{f}: \mathbb{R}^{3,1} \rightarrow C l_{3,1}$ of a (16 dimensional) spacetime algebra $C l_{3,1}$ module function $f \in L^{2}\left(\mathbb{R}^{3,1} ; C l_{3,1}\right)$ with spacetime vectors $\mathbf{x}=t \mathbf{e}_{0}+\vec{x} \in \mathbb{R}^{3,1}, \vec{x}=x \mathbf{e}_{1}+y \mathbf{e}_{2}+z \mathbf{e}_{3} \in \mathbb{R}^{3}$, and spacetime frequency vectors $\mathbf{u}=s \mathbf{e}_{0}+\vec{u} \in \mathbb{R}^{3,1}, \vec{u}=u \mathbf{e}_{1}+v \mathbf{e}_{2}+w \mathbf{e}_{3} \in \mathbb{R}^{3}$ is defined by

$$
\stackrel{\diamond}{f}(\mathbf{u})=\int_{\mathbb{R}^{3,1}} e^{-\mathbf{e}_{0} t s} f(\mathbf{x}) e^{-i_{3} \vec{x} \cdot \vec{u}} d^{4} \mathbf{x},
$$

with $d^{4} \mathbf{x}=d t d x d y d z$.

Because of (66) definition [5.3 is fully compatible with definition [5.1 since (70) is nothing but a (right) linear combination of (59). To show this, we can use $C l_{3,1} \cong V_{t} \otimes C l_{3,0}^{+}$or we can e.g. rewrite a general spacetime algebra module function $f \in L^{2}\left(\mathbb{R}^{3,1} ; C l_{3,1}\right)$ as a (right) linear combination of four volume-time subalgebra module $L^{2}\left(\mathbb{R}^{3,1} ; V_{t}\right)$ functions

$$
\begin{aligned}
& f=f_{s}+f_{1} \mathbf{e}_{1}+f_{2} \mathbf{e}_{2}+f_{3} \mathbf{e}_{3}+f_{23} \mathbf{e}_{2} \mathbf{e}_{3}+f_{31} \mathbf{e}_{3} \mathbf{e}_{1}+f_{12} \mathbf{e}_{1} \mathbf{e}_{2}+f_{123} i_{3}+f_{0} \mathbf{e}_{0}+ \\
& +f_{01} \mathbf{e}_{0} \mathbf{e}_{1}+f_{02} \mathbf{e}_{0} \mathbf{e}_{2}+f_{03} \mathbf{e}_{0} \mathbf{e}_{3}+f_{023} \mathbf{e}_{0} \mathbf{e}_{2} \mathbf{e}_{3}+f_{031} \mathbf{e}_{0} \mathbf{e}_{3} \mathbf{e}_{1}+f_{012} \mathbf{e}_{0} \mathbf{e}_{1} \mathbf{e}_{2}+f_{4} i_{4} \\
& \quad=f_{s}+f_{0} \mathbf{e}_{0}+f_{123} i_{3}+f_{4} i_{4}+\left\{f_{1}+f_{01} \mathbf{e}_{0}+f_{23} i_{3}+f_{023} i_{4}\right\} \mathbf{e}_{1}+ \\
& \quad+\left\{f_{2}+f_{02} \mathbf{e}_{0}+f_{31} i_{3}+f_{031} i_{4}\right\} \mathbf{e}_{2}+\left\{f_{3}+f_{03} \mathbf{e}_{0}+f_{12} i_{3}+f_{012} i_{4}\right\} \mathbf{e}_{3} .
\end{aligned}
$$

The four $L^{2}\left(\mathbb{R}^{3,1} ; V_{t}\right)$ functions of (71) are $\left\{f_{s}+f_{0} \mathbf{e}_{0}+f_{123} i_{3}+f_{4} i_{4}, f_{1}+f_{01} \mathbf{e}_{0}+\right.$ $\left.f_{23} i_{3}+f_{023} i_{4}, f_{2}+f_{02} \mathbf{e}_{0}+f_{31} i_{3}+f_{031} i_{4}, f_{3}+f_{03} \mathbf{e}_{0}+f_{12} i_{3}+f_{012} i_{4}\right\}$, where all 16 coefficient functions $\left\{f_{s}, f_{0}, f_{1}, \cdots, f_{4}\right\}$ belong to $L^{2}\left(\mathbb{R}^{3,1}, \mathbb{R}\right)$.

Because of (68) the general SFT of Clifford module $L^{2}\left(\mathbb{R}^{3,1} ; C l_{3,1}\right)$ functions of definition 5.3 is also invertible

$$
f(\mathbf{x})=\frac{1}{(2 \pi)^{4}} \int_{\mathbb{R}^{3,1}} e^{\mathbf{e}_{0} t s} \stackrel{\diamond}{f}(\mathbf{u}) e^{i_{3} \vec{x} \cdot \vec{u}} d^{4} \mathbf{u} .
$$

\subsection{SFT of $f_{ \pm}$split parts and physical interpretation}

Further application of analogous (right) linearity arguments also yield that the split (61) and (62) can also be applied to general multivector Clifford module functions $f \in L^{2}\left(\mathbb{R}^{3,1} ; C l_{3,1}\right)$. In (61) and (62) we can thus simply replace the $L^{2}\left(\mathbb{R}^{3,1} ; V_{t}\right)$ functions by $L^{2}\left(\mathbb{R}^{3,1} ; C l_{3,1}\right)$ function 19 . This carries on to the general SFTs of the split functions $f_{ \pm}$, which are formally identical to (63) if we again replace the $L^{2}\left(\mathbb{R}^{3,1} ; V_{t}\right)$ functions by $L^{2}\left(\mathbb{R}^{3,1} ; C l_{3,1}\right)$ functions.

\footnotetext{
${ }^{18} \mathrm{Cl}_{3,1}$ is also isomorphic to the tensor product $V_{t} \otimes C l_{3,0}^{+}$, with $V_{t}$ defined as in section 5.1 and $\mathrm{Cl}_{3,0}^{+}$defined as in section 1.1 (See [21], sections 4.1 and 4.2.)

${ }^{19}$ Again the $f_{ \pm}$split (62) solely depends on the choice of time direction $\mathbf{e}_{0}$.
} 
We can therefore rewrite the SFT (70) for $f \in L^{2}\left(\mathbb{R}^{3,1} ; C l_{3,1}\right)$ as

$$
\begin{gathered}
\stackrel{\diamond}{f=\grave{f}_{+}}+\stackrel{\diamond_{f}}{-}=\int_{\mathbb{R}^{3,1}} f_{+} e^{-i_{3}(\vec{x} \cdot \vec{u}-t s)} d^{4} x+\int_{\mathbb{R}^{3,1}} f_{-} e^{-i_{3}(\vec{x} \cdot \vec{u}+t s)} d^{4} x \\
=\int_{\mathbb{R}^{3,1}} e^{-\mathbf{e}_{0}(t s-\vec{x} \cdot \vec{u})} f_{+} d^{4} x+\int_{\mathbb{R}^{3,1}} e^{-\mathbf{e}_{0}(t s+\vec{x} \cdot \vec{u})} f_{-} d^{4} x .
\end{gathered}
$$

Complex spacetime Fourier transformations, with $\exp \{-i(\vec{x} \cdot \vec{u}-t s)\}$ (where $i \in \mathbb{C}$ ) as the related complex kernel, are e.g. used for electromagnetic fields in spatially dispersive media 22] or in electromagnetic wavelet theory [23].

In physics $f_{+}$can be interpreted as (time dependent) multivector amplitude of a rightward (forward) moving wave packet, and $f_{-}$as that of a leftward (backward) moving wave packet. But we emphasize that both the non-commutative multivector structure and the geometric interpretation (e.g. of $i_{3}$ as oriented 3D spatial volume trivector) go beyond conventional treatment.

We get the consequent generalization of theorem 4.2 , i.e. the $G L\left(\mathbb{R}^{3,1}\right)$ transformation properties of the SFT in the form of

Theorem $5.4\left(G L\left(\mathbb{R}^{3,1}\right)\right.$ transformation properties of the SFT). The SFT of a $C l_{3,1}$ module function $f \in L^{2}\left(\mathbb{R}^{2} ; C l_{3,1}\right)$ with a $G L\left(\mathbb{R}^{3,1}\right)$ transformation $\mathcal{A}$ of its vector argument is given by

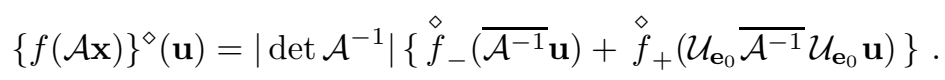

This concludes our brief example of a higher dimensional multivector generalization of the QFT for $L^{2}\left(\mathbb{R}^{2} ; \mathbb{H}\right)$ functions to a SFT for $L^{2}\left(\mathbb{R}^{3,1} ; C l_{3,1}\right)$ functions. We again emphasize that mathematically many other generalizations are in fact possible and we expect a number of them to be of great utility in applications.

\section{Conclusions}

We employed a convenient rewriting of quaternions only in terms of $\boldsymbol{i}$ and $\boldsymbol{j}$, keeping one to the left and the other to the right; and a quaternion split, which in spacetime applications is closely related the choice of the time direction. This allowed us to investigate a range of properties of the QFT, last but not least the behavior of the QFT under general linear automorphisms.

General coordinate free formulation in combination with quaternion to Clifford subalgebra isomorphisms opens the door to a wide range of QFT generalizations. These non-commutative multivector Fourier transforms act on functions from $\mathbb{R}^{m, n}, m, n \in \mathbb{N}_{0}$ to Clifford geometric algebras $C l_{m, n}$ (or appropriate subalgebras). We demonstrated this by establishing two multivector Fourier transforms: the volume-time and the spacetime Fourier transforms. They await application, e.g. in the fields of dynamic fluid and gas flows, seismic analysis, to electromagnetic phenomena, in short wherever spatial data are recorded with time. We expect other generalizations of the QFT obtained by the same methods to be of great potential use as well. 


\section{Acknowledgements}

I thank God, the Creator: How great are your works, O LORD, how profound your thoughts! 24]. I thank my family for their total loving support, and B. Mawardi, A. Hayashi and O. Yasukura for helpful comments. I thank the editor S. Krausshar, and the anonymous referees for helpful suggestions.

\section{References}

[1] D. Hestenes, G. Sobczyk, Clifford Algebra to Geometric Calculus, Kluwer, 1984.

[2] T. Needham, Visual Complex Analysis, Oxford University Press, 2001. O. Rodrigues, Des lois géométriques qui régissent des déplacements d'un système solide, Journal de Mathématiques Pures et Appliquées (Liouville), Vol. 5 (1840) p. 380.

[3] F. Brackx, R. Delanghe, and F. Sommen, Clifford Analysis, Vol. 76 of Research Notes in Mathematics, Pitman Advanced Publishing Program, 1982.

[4] T. Bülow, M. Felsberg and G. Sommer, Non-commutative Hypercomplex Fourier Transforms of Multidimensional Signals, in G. Sommer (ed.), Geom. Comp. with Cliff. Alg., Theor. Found. and Appl. in Comp. Vision and Robotics, Springer (2001), 187-207.

[5] C. Li, A. McIntosh and T. Qian, Clifford Algebras, Fourier Transform and Singular Convolution Operators On Lipschitz Surfaces, Revista Matematica Iberoamericana, 10 (3), (1994), 665-695.

[6] A. McIntosh, Clifford Algebras, Fourier Theory, Singular Integrals, and Harmonic Functions on Lipschitz Domains, chapter 1 of J. Ryan (ed.), Clifford Algebras in Analysis and Related Topics, CRC Press, Boca Raton, 1996.

[7] T. Qian, Paley-Wiener Theorems and Shannon Sampling in the Clifford Analysis Setting in R. Ablamowicz (ed.), Clifford Algebras - Applications to Mathematics, Physcis, and Engineering, Birkäuser, Basel, (2004), 115-124.

[8] J. Ebling and G. Scheuermann, Clifford Fourier Transform on Vector Fields, IEEE Transactions on Visualization and Computer Graphics, 11 (4), July/August (2005), 469-479.

[9] B. Mawardi, E. Hitzer, Clifford Fourier Transformation and Uncertainty Principle for the Clifford Geometric Algebra $C l_{3,0}$, AACA, 16(1) (2006), 41-61.

[10] E. Hitzer, B. Mawardi, Uncertainty Principle for the Clifford Geometric Algebra $C l_{n, 0}, n=3(\bmod 4)$ based on Clifford Fourier transform, in Springer SCI book series Applied and Numerical Harmonic Analysis, 2006, pp. 45-54.

[11] E. Hitzer, B. Mawardi, Clifford Fourier Transform on Multivector Fields and Uncertainty Principles for Dimensions $n=2(\bmod 4)$ and $n=3(\bmod 4)$, preprint for ICCA7 proceedings.

[12] T. Bülow, Hypercomplex Spectral Signal Representations for the Processing and Analysis of Images, PhD Thesis, Univ. of Kiel, 1999.

[13] T.A. Ell, Quaternion-Fourier Transforms for Analysis of Two-Dimensional Linear Time-Invariant Partial Differential Systems, in Proc. of the 32nd Conf. on Decision and Control, IEEE (1993), 1830-1841. 
[14] M. Felsberg, Low-Level Image Processing with the Structure Multivector, PhD thesis, Univ. of Kiel, 2002.

[15] S.C. Pei, J.J. Ding, J.H. Chang, Efficient Implementation of Quat. Fourier Transf., Convolution, and Correlation by 2-D Complex FFT, IEEE Trans. on Sig. Proc.49(11) (2001), 2783-2797.

[16] F. Catoni, R. Cannata, P. Zampeti, An Introduction to Commutative Quaternions AACA 16(1) (2006), 1-28.

[17] D. Hestenes, New Foundations for Classical Mechanics, Kluwer, 1999.

[18] H.S.M. Coxeter, W.O.J. Moser, Generators and Relations for Discrete Groups, Springer, 4th ed., 1980.

[19] E. Cartan, La géométrie des groupes simples, Ann. Mat. Pura Appl. (4) (1927), 209256. Complément au mémoire sur la géométrie des groupes simples, Ann. Mat. Pura Appl. (4), 5 (1928), 253-260.

[20] D. Hestenes, Space-Time Algebra, Gordon and Breach, 1966.

[21] P. Girard, Quaternions, algèbre de Clifford et physique relativiste, Presses polytechniques, et universitaires romandes, 2004.

[22] G. Agarwal, D. Pattanayak, E. Wolf, Structure of the Electromagnetic Field in a Spatially Dispersive Medium, Phys. Rev. Lett. 27 (1971), 1022-1025.

[23] G. Kaiser, Physical wavelets and their sources: Real physics in complex spacetime, preprint, arxiv.org/abs/math-ph/0303027

[24] Psalm 92, verse 5, New Int. Version of the Bible, www.biblegateway.com

Eckhard M. S. Hitzer

Department of Applied Physics

University of Fukui

3-9-1 Bunkyo

910-8507 Fukui

Japan

e-mail: hitzer@mech.fukui-u.ac.jp 